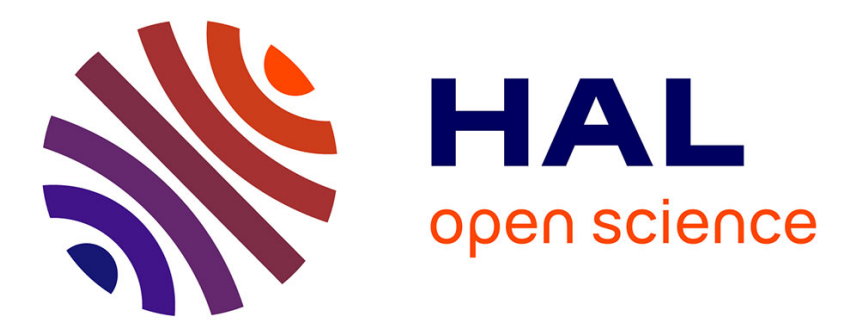

\title{
The Helium Focusing Cone of the Local Interstellar Medium Close to the Sun
}

J. G. Michels, J. C. Raymond, Jean-Loup Bertaux, Eric Quémerais, Rosine Lallement, Y.-K. Ko, D. Spadaro, L. D. Gardner, S. Giordano, R. O’Neal, et al.

\section{To cite this version:}

J. G. Michels, J. C. Raymond, Jean-Loup Bertaux, Eric Quémerais, Rosine Lallement, et al.. The Helium Focusing Cone of the Local Interstellar Medium Close to the Sun. The Astrophysical Journal, 2002, 568 (1), pp.385. 10.1086/338764 . insu-02902316

\section{HAL Id: insu-02902316 https://hal-insu.archives-ouvertes.fr/insu-02902316}

Submitted on 18 Jul 2020

HAL is a multi-disciplinary open access archive for the deposit and dissemination of scientific research documents, whether they are published or not. The documents may come from teaching and research institutions in France or abroad, or from public or private research centers.
L'archive ouverte pluridisciplinaire HAL, est destinée au dépôt et à la diffusion de documents scientifiques de niveau recherche, publiés ou non, émanant des établissements d'enseignement et de recherche français ou étrangers, des laboratoires publics ou privés. 


\title{
THE HELIUM FOCUSING CONE OF THE LOCAL INTERSTELLAR MEDIUM CLOSE TO THE SUN
}

\author{
J. G. Michels, ${ }^{1,2}$ J. C. Raymond,${ }^{1}$ J. L. Bertaux,${ }^{3}$ E. Quémerais, ${ }^{3}$ R. Lallement,${ }^{3}$ Y.-K. Ko, ${ }^{1}$ D. Spadaro, ${ }^{4}$ L. D. Gardner, ${ }^{1}$ \\ S. Giordano, ${ }^{5}$ R. O’Neal, ${ }^{1}$ S. Fineschi, ${ }^{5}$ J. L. Kohl, ${ }^{1}$ C. Benna, ${ }^{5}$ A. Ciaravella, ${ }^{1}$ M. Romoli, ${ }^{6}$ and D. Judge ${ }^{7}$ \\ Received 2001 August 3; accepted 2001 November 19
}

\begin{abstract}
The Solar and Heliospheric Observatory (SOHO) Ultraviolet Coronagraph Spectrometer is used to observe the interplanetary He focusing cone within 1 AU. Taken over $2 \mathrm{yr}$ and from differing orbit positions, the series of observations includes measurements of He I $584 \AA$ and Ly $\beta$ intensities. The cone itself is spatially well defined, and the He I intensity within the cone was $\sim 45 \mathrm{R}$ in 1996 December, compared with $\sim 1 \mathrm{R}$ for lines of sight outward from 1 AU. Between 1996 December and 1998 June, the focusing cone dimmed by a factor of 3.3 as the level of solar activity rose. This is the first time that interstellar helium is observed so near the Sun. Measured intensities are compared to a detailed temperature and density model of interstellar helium in the solar system. The model includes EUV ionization but does not include ionization by electron impact from solar wind electrons. There are several features in the data model comparison that we attribute to the absence of electron impact ionization in the model. The absolute maximum intensity of $45 \mathrm{R}$ first measured in 1996 December calls for an ionization $45 \%$ more intense than the EUV photoionization alone as measured by the Solar EUV Monitor/Charge, Element, and Isotope Analysis System (SEM/CELIAS) on SOHO. Important day-to-day variations of the intensity are observed, as well as a general decrease as the solar activity rises (both absolute and divided by a model with a constant ionization). This general decrease is even larger than predicted by a model run with the SEM/CELIAS photoionization rate alone, in spite of a factor of 1.5 increase of this rate from 1996 December to 1998 June. At this time, an additional ionization rate of $0.56 \times 10^{-7} \mathrm{~s}^{-1}$ (compared with $1.00 \times 10^{-7} \mathrm{~s}^{-1}$ from solar EUV) is required to fit the measured low intensity. We attribute this additional rate to solar wind electron impact ionization of the atoms. This shows that the helium intensity pattern is a very sensitive indicator of the electron density and temperature near the Sun.

Subject headings: interplanetary medium — solar wind — ultraviolet: solar system
\end{abstract}

\section{INTRODUCTION}

Much as a powerful lighthouse lamp illuminates individual water droplets as it shines through a thick fog, light from the Sun is scattered from the neutral hydrogen and helium atoms of the interstellar medium (ISM). Solar ultraviolet light is resonantly absorbed by the neutrals, which then reemit ultraviolet photons. The resulting resonant emission imparts a weak UV glow to the sky that is readily detectable. Indeed, much of what we know about the very nearby ISM comes from observations of the region in space surrounding the Sun, often referred to as the local interstellar medium (LISM; Bertaux et al. 1997; Bertaux \& Blamont 1971). Study of the LISM is pursued by both astronomers seeking to probe the structure of the ISM and physicists intent on characterization of solar activity and the solar wind.

While the acronym LISM generally designates the ISM in the vicinity of the Sun (say, within 15 pc from the Sun), the interstellar gas inside the solar system belongs to a particular parcel of interstellar gas called the Local Interstellar Cloud (LIC; Lallement \& Bertin 1992; Frisch 1998). This particular cloud in which the Sun is presently embedded was

\footnotetext{
${ }^{1}$ Harvard-Smithsonian Center for Astrophysics, Cambridge, MA 02138 .

2 Princeton Materials Institute, Princeton University, 70 Prospect Avenue, Princeton, NJ 08540.

${ }^{3}$ Centre National de la Recherche Scientifique, Service d'Aéronomie, BP3, 91371 Verrières le Buisson Cedex, France.

${ }^{4}$ Osservatorio Astrofisico di Catania, I-95125 Catania, Italy.

${ }^{5}$ Osservatorio di Torino, I-10025 Pino Torinese, Italy.

${ }^{6}$ Università di Firenze, I-50125 Firenze, Italy.

${ }^{7}$ Space Science Center, University of Southern California, Los Angeles, CA 90089.
}

identified by Doppler triangulation of interstellar absorption lines toward a number of nearby stars. It was found that the Sun is moving at $26 \mathrm{~km} \mathrm{~s}^{-1}$ through the LIC in a direction different from the motion of the Sun toward the apex, which is defined in the local standard of rest (LSR) with respect to the nearby stars. This is because the LIC is itself moving with respect to the LSR, and hence the name of interstellar wind, or breeze, given its moderate velocity.

Roiling the interstellar breeze is the solar wind streaming forth from the Sun, which disrupts the ISM through radiation, ionization, and its own prodigious gravitational attraction. As the Sun churns through the ISM, it creates a cavity largely devoid of neutral hydrogen, while at the same time collecting in its wake a concentrated cloud of neutral helium atoms. The He density enhancement has been labeled the helium focusing cone because of its conelike shape, with base near the Sun and peak pointing opposite the direction of the incoming breeze. The focusing cone is a consequence of the Sun's gravitational attraction of neutral helium atoms from the ISM. Although dimensions of the focusing cone depend on the inflow velocity and temperature of the neutral gas, the core generally extends from the Sun with significant density enhancements out past 1 AU. Lined up with the directional vector of the interstellar breeze, the angular coordinates of the cone axis are $-5.6 \pm 0.4$ latitude below the ecliptic and $73.9 \pm 0.8$ ecliptic longitude (Witte, Banaszkiewicz, \& Rosenbauer 1996). The density of helium atoms within the focusing cone is a function of solar photoionization and collisional ionization rates.

For hydrogen atoms swept in by the interstellar wind, the situation is nearly reversed from that of helium. Radiation pressure caused by the scattering of solar Ly $\alpha$ photons acts 
as an outward force. While the ratio of solar radiation pressure to gravitational force is negligible for helium, it varies between $\simeq 0.5$ and 1.5 for hydrogen. In addition, hydrogen is subject to rapid ionization by charge exchange with solar wind protons, which is the dominant ionization process for H. Because of hydrogen's high ionization rate and solar radiation pressure, a cavity with an extremely low density of neutral hydrogen atoms is carved out of the ISM both upwind and, to a greater extent, downwind from the Sun.

Most probes of the interstellar gas in the heliosphere have relied on observations of backscattered solar resonant radiation. Other methods for characterizing the ISM include direct measurements of neutral interstellar helium (Witte et al. 1993) and measurements of pickup ions (Möbius et al. 1995). Pickup ion measurements detect ions produced from the neutral gas through ionization by solar UV, electron impact, or charge exchange with the solar wind. They are discriminated from solar wind ions by their peculiar velocity distribution, providing an accurate account of the helium density (Gloeckler \& Geiss 1998). An important point is the excellent agreement between the velocity vector and the temperature of the local interstellar cloud mentioned above and the corresponding parameters for the flow of interstellar helium in the heliosphere derived from the Ulysses particle data (Witte et al. 1996). This is expected, since according to models the helium flow is unaffected by interaction with the plasma at the heliospheric interface, i.e., where the interstellar ionized gas encounters the solar wind (Bleszynski 1987).

Launch of the Solar and Heliospheric Observatory (SOHO) satellite in 1995 December to an orbit about the L1 libration point delivered several optical instruments capable of observing the LISM with high spectral and spatial resolution. Among these instruments is the Ultraviolet Coronagraph Spectrometer (UVCS), which is designed to study the nature of the solar corona and generation of the solar wind (Kohl et al. 1995). SOHO/UVCS can measure emission intensities of less than $1 \mathrm{R}$. It is currently the only spaceborne instrument capable of observing $\mathrm{He}$ I intensities well inside $1 \mathrm{AU}$, making $\mathrm{SOHO} / \mathrm{UVCS}$ ideally suited for study of the helium focusing cone.

In addition, the Solar Wind Anisotropies (SWAN) instrument aboard $\mathrm{SOHO}$ has mapped the whole sky distribution of $\mathrm{Ly} \alpha$ emission from $\mathrm{H}$ atoms resonantly illuminated by the Sun (Bertaux et al. 1997). Large differences are observed when comparing maps made in June and December. In both cases, a maximum of hydrogen emission is observed in the hemisphere where the interstellar wind enters, while minimum hydrogen intensity exists downstream from the Sun. In June, the Earth is upwind and very near to the region of maximal hydrogen emissivity, while in December the Sun stands between the source of the interstellar wind and the Earth. Accordingly, the distance to the maximum source of light is increased and its angular size is decreased. Comparing maps at $1 \mathrm{yr}$ intervals reveals slight differences as a result of changes in the latitude distribution of the solar wind as the solar cycle progresses and subsequent modification of the destruction pattern of interstellar $\mathrm{H}$ atoms by charge exchange with solar wind protons. Complementary $\mathrm{SOHO/}$ UVCS measurements of the Ly $\alpha$ profile exist and will be analyzed in a separate paper (D. Spadaro et al. 2002, in preparation).

In this paper we report an increase by over a factor of 5 in the He I $584 \AA$ emission intensity backscattered from helium atoms as measured by $\mathrm{SOHO}$ /UVCS within the focusing cone are compared to regions outside the cone. Measurements of the focusing cone over several successive days characterize the sharp spatial extent of the cone and may reveal changes in neutral helium density with different heliocentric height. From 1996 until 1998, as solar activity increased with the progression toward solar maximum, the brightness of the He focusing cone declined by a factor of 2 . The brightness of the solar disk in the He I $584 \AA$ line must have increased somewhat with solar activity during that time, so the neutral helium density decreased by a larger factor.

\section{INSTRUMENT DESCRIPTION}

$\mathrm{SOHO} / \mathrm{UVCS}$ has been previously described by Kohl et al. (1995). Therefore, only a brief description is provided with special emphasis on the instrument's performance relative to second-order spectral lines. Designed to observe the solar corona between 1.2 and $12 R_{\odot}$, UVCS has two ultraviolet spectral channels. The Ly $\alpha$ channel covers the range 1150-1350 $\AA$, while the O vi channel is optimized for measurements of the $\mathrm{O}$ vi lines at 1032 and $1037 \AA$ and covers the range 945-1123 $\AA$ (473-561 $\AA$ in second order). Use of a convex mirror inserted in the $\mathrm{O}$ vi channel provides a redundant optical path for Ly $\alpha$ allowing for the focusing of spectral lines in the range 1160-1270 $\mathrm{\AA}(580-635 \AA$ in second order) on the $\mathrm{O}$ VI detector. For $\mathrm{SOHO} / \mathrm{UVCS}$ observations of the LISM, we relied exclusively on the $\mathrm{O}$ vi detector and captured the He I line using the redundant path. The optical elements are a telescope mirror ( $\mathrm{SiC}$ coated), a 3600 line $\mathrm{mm}^{-1}$ toric diffraction grating (iridium coated) and an XDL detector ( $\mathrm{KBr}$ coated).

Conversion from count rates to intensities in rayleighs is a function of the effective area of the telescope mirror, the solid angle subtended by the UVCS field of view, and the time of exposure. The $\mathrm{SOHO} / \mathrm{UVCS}$ telescope is externally and internally occulted, with the exposed telescope area dependent on the heliographic height of the observation. The amount of internal vignetting is controlled by an internal occulter that can be moved across the telescope mirror to block any light diffracted by the external occulter that would otherwise be imaged onto the slits. Moving the internal occulter alters the width of the exposed primary mirror. In order to minimize the amount of solar-disk stray light and ensure that recorded intensities are solely from the LISM, the instrument was configured to occult slightly more of the mirror than strictly required by geometrical optics. This setting translates into slightly less mirror area. The effective vignetting as a function of internal occulter position has been verified in flight.

\section{RADIOMETRIC CALIBRATION}

The He I $\lambda 584$ line was observed in the UVCS O vi channel in second order using the "redundant" path. While no direct end-to-end efficiency measurements have been made at this wavelength, measurements of components and/or replicas of components exist and allow an estimation, albeit with significant uncertainty, of the end-to-end response. Details of this estimation follow.

The UVCS radiometric calibration in first order was determined by laboratory measurements in 1995 June (Gardner et al. 1996; Kohl et al. 1995) at a "standard aper- 
ture" of $11 \mathrm{~mm}$. The end-to-end efficiency of the UVCS $\mathrm{O}$ vi channel was measured to be $0.0035( \pm 15 \%)$ at the 104.8 $\mathrm{nm}$ line of Ar I. The behavior of the flight grating for apertures greater than and less than the standard one has been taken from measurements in the laboratory on a grating replicated from the same master as the flight grating (Gardner et al. 2000). UVCS spectra of $\tau$ Tau (B3 V) are in agreement with Voyager UVS spectra to better than $15 \%$ at wavelengths between 970 and $1070 \AA$. An even stronger confirmation of the in-flight performance is provided by comparison of the UVCS spectrum of the sdO standard star Feige 110 obtained in 2001 March with the spectrum obtained with the Far Ultraviolet Spectroscopic Explorer (FUSE). Again, the two agree to within about $\pm 15 \%$. The constancy of first-order $\mathrm{O}$ VI channel response over time has been confirmed by observing several stars at $1 \mathrm{yr}$ intervals. No change has been seen in the count rates of bright stars such as $\delta$ Sco. We believe the calibration uncertainty to be less than $\pm 20 \%(1 \sigma)$ for first-order lines.

Based on measurements of the individual components and/or replicas of the individual components, the efficiency at $104.8 \mathrm{~nm}$ should be the product of the reflectivity of the SiC-coated telescope mirror (46\%; Osantowski et al. 1991), the grating reflectivity $(18 \%)$ and the groove efficiency averaged over the "standard aperture" of $11 \mathrm{~mm}(49 \%)$ (unpublished measurements in our laboratory), and the quantum efficiency of the $\mathrm{KBr}$-coated XDL detector measured as a component before instrument assembly (18\%; Siegmund et al. 1994; Kohl et al. 1995). The product of these numbers is $0.73 \%$, however, 2.1 times the measured end-to-end value. Similar analysis has been carried out for H I Ly $\alpha 121.6 \mathrm{~nm}$, which in the UVCS O VI channel includes an $85^{\circ}$ angle of incidence reflection ( $75 \%$ efficient) off the $\mathrm{MgF} 2 / \mathrm{Al}$-coated "redundant path" mirror. Here the difference factor is 3.2. Given the nature of the optical components and the environmental conditions experienced by UVCS, we believe the loss in efficiency at both wavelengths is consistent with prelaunch damage to the $\mathrm{KBr}$ coating of the detector from exposure to water vapor (i.e., humidity). One approach to model the loss in efficiency is to assume that exposure to humidity concentrates the $\mathrm{KBr}$ on certain regions of the channel plate and leaves other regions bare. In that case, the overall efficiency of the degraded detector could be described as a linear combination of the efficiencies of bare microchannel plates from the same lot as UVCS (Siegmund et al. 1995) and the measured and assumed undegraded $\mathrm{KBr}$-coated microchannel plate (i.e., the actual UVCS detector before any degradation; Kohl et al. 1995). If all of the degradation of the UVCS system calibration is attributed to the detector, then $73 \%$ "bare" plus $27 \% \mathrm{KBr}$ will provide the observed degradation at both 104.8 and 121.6 nm.

To obtain an efficiency at $\mathrm{He}$ I $58.4 \mathrm{~nm}$, we first modify the efficiency of the XDL detector using the coefficients deduced above. The detector efficiency at $58.4 \mathrm{~nm}$ is then reduced from $27 \%$ to $12 \%$. Multiplying by the component efficiencies at $58.4 \mathrm{~nm}$ (telescope mirror: $27 \%$; average grating efficiency for the first $11 \mathrm{~mm}: 4.6 \%$; redundant mirror reflectivity: $70 \%$ ) then provides an end-to-end efficiency of about 0.0010 .

An alternative method of estimating the efficiency at 58.4 $\mathrm{nm}$ can be made based on the performance of a "life-test" laboratory KBr-coated detector. Jelinsky, Seigmund, \& Mir (1996) report efficiency measurements that have been made periodically for 67 months. The detector, which was stored in dry nitrogen between measurement updates, has shown a $30 \%$ loss of quantum efficiency at $58.4 \mathrm{~nm}$, a $21 \%$ loss at $104.8 \mathrm{~nm}$, and a loss of $36 \%$ at $121.6 \mathrm{~nm}$ over the 67 months. Admittedly, the environment experienced by the UVCS detectors has been very different from that experienced by the laboratory one. Indeed, it has likely been better controlled and more benign than that experienced by UVCS during integration and testing. Nevertheless, if one assumes that the UVCS O VI detector has degraded in the same relative proportions as the life-test detector, then the degradation at $58.4 \mathrm{~nm}$ can be related to the observed degradations at 104.8 and $121.6 \mathrm{~nm}$. Thus, comparing to $104.8 \mathrm{~nm}$, one obtains a degradation given by the ratio of the test detector's degradation at 58.4 and at $104.8(0.30 / 0.21)$ times the observed degradation of the UVCS detector at $104.8 \mathrm{~nm}$. The expected UVCS end-to-end efficiency at $58.4 \mathrm{~nm}$ is 0.00061 . Comparing instead to $121.6 \mathrm{~nm}$, one obtains a degradation given by the ratio of the test detector's degradation at 58.4 and at $121.6 \mathrm{~nm}(0.30 / 0.36)$, times the observed degradation of the UVCS detector at $121.6 \mathrm{~nm}$, and the expected UVCS end-to-end efficiency at $58.4 \mathrm{~nm}$ is 0.00052 .

Lacking better information, we choose to average algebraically the three values $(0.0010,0.00061$, and 0.00052$)$ to obtain a best estimate of 0.00071 . The estimated uncertainty is $50 \%$ of this number and encompasses all three values.

In order to determine the $\mathrm{He}$ I radiances for this paper, additional corrections associated with the details of the illumination of the grating and redundant mirror must be made also. In order to keep disk-originating stray light to a minimum, different telescope mirror (and hence grating) apertures are used for observations at different heliocentric heights. The effective area of UVCS is not simply aperture proportional because of vignetting effects associated with the redundant mirror and because of efficiency variations across the grating's surface (Gardner et al. 2000). For example, for the position of the $\mathrm{He} \mathrm{I}$ line on the detector at all apertures, some of the light misses the redundant mirror, a relatively large effect at small (below $3 R_{\odot}$ ) apertures, and at large apertures (above $6 R_{\odot}$ ) the redundant mirror is "overfilled," resulting in some additional loss of light. There is also some variation in efficiency across the grating, as measured in the laboratory. Measurements in flight using $\mathrm{H} \mathrm{I}$ Ly $\alpha$ as a function of position on the detector and internal occulter position (aperture) show that the relative efficiencies at 5,7 , and $9 R_{\odot}$ are $0.65,1.0$, and 0.73 .

Overall, we believe that the uncertainty in the He I fluxes is about $\pm 50 \%$. Relative fluxes should be reliable to about $10 \%$ except for a few observations affected by high background or short exposure times.

\section{OBSERVATIONS}

Between 1996 December and 1998 June, we performed a series of long integrations using grating positions where the redundant mirror captures the He I $584 \AA$ line in second order, and the direct illumination first-order range of $\sim 1037-945 \AA$ prominently includes $\operatorname{Ly} \beta$ and very faint emission from O VI $1032 \AA$ and $C_{\text {III }} 977 \AA$. For all but three of the observations, UVCS was configured to look above the south coronal hole at heliocentric heights between 5 and $9 R_{\odot}$. Directing the UVCS to look at the coronal holes minimized coronal emission, thus leaving less scattered light to corrupt our intensity measurements of the LISM's resonant 
glow, and the southern position places the line of sight close to the axis of the focusing cone.

The halo orbit of $S O H O / U V C S$ about the L1 Lagrangian point keeps the spacecraft always between the Earth and the Sun. As a result of the Earth's orbital motion, observations at different times of the year place $\mathrm{SOHO} / \mathrm{UVCS}$ in varying positions relative to the focusing cone that collects on the downwind side of the Sun at $74^{\circ}$ ecliptic longitude (Witte et al. 1996). In early December, $\mathrm{SOHO}$ /UVCS looks into the wind toward the Sun and therefore through the focusing cone. The UVCS line of sight intersects the axis of the focusing cone at $0.19,0.25$, and 0.30 AU for observations directed at heliocentric heights of 5,7 , and $9 R_{\odot}$. Six months later in June, the interstellar wind is on the back of UVCS. The UVCS line of sight intersects the focusing cone on the far side of the Sun at 0.75 AU for an observation of the coronal hole at $9 R_{\odot}$. The He I intensity recorded in June is therefore expected to be less than in December, as the UVCS line of sight intersects the cone at a distance further away from the Sun where the helium density is less. The effective path length in June is much longer than in December, however, as the UVCS line of sight looks along the cone rather than through it. Thus, the difference in intensities is smaller than would be expected from where the line of sight crosses the focusing cone.

The SOHO/UVCS data from the focusing cone observations are presented in Table 1, which contains the date of the observation, $\mathrm{SOHO}$ 's position in heliocentric latitude and longitude, UVCS pointing and position angle, and intensities for $\mathrm{He} \mathrm{I}, \mathrm{Ly} \beta$, and $\mathrm{C}$ III. The $\mathrm{C}$ III line is important as an indication of the instrumental and F-corona contribution to the $\mathrm{He}$ I and $\mathrm{Ly} \beta$ fluxes. At coronal temperatures, there is no C III emission, and no local interstellar component is expected. The $\mathrm{SOHO}$ position is included in Table 1 because the halo orbit offsets the spacecraft from the EarthSun line. As a result, the position of the Sun with respect to the background stars is shifted by as much as $15^{\prime}$. This shift will have only a small effect on the He I brightness.

As a sample of the data quality, we show two of the observations in Figure 1. The 1996 December 5 observation was a 6 hour integration, and the 1997 December 2 observation was 9 hours. The two wavelength scales for each spectrum correspond to direct illumination and to illumination by the redundant mirror. Reflection from the redundant mirror reverses the direction of increasing wavelength on the detector. The position of the direct illumination C III $\lambda 977$ line relative to the redundant path $\mathrm{He}$ I $\lambda 584$ line depends on the grating position. Both spectra are sums over the length of the spectrograph slit. The background is largely scattered Ly $\alpha$ photons distributed over the entire detector. The decrease in the He I intensity between 1996 and 1997 is readily apparent even with the different exposure times. The relative uncertainty in the measurements is dominated by the uncertainty in placement of the (somewhat uneven) continuum. It is also necessary to separate the He I signal from a faint ghost of Ly $\alpha$ located at $1169.8 \AA$. The ghost typically provides only about $10 \%$ as many counts as the He I line except when the helium line is faint (1997 December and 1998 June) and when the coronal Ly $\alpha$ line makes a strong contribution (1997 August and exposures at $5 R_{\odot}$ ). The position of the ghost was determined from calibration measurements with the internal occulter pulled back and was verified by comparison with the symmetric ghost on the long-wavelength side of Ly $\alpha$. The short exposures and low fluxes of the 1997 December observations tend also to increase the uncertainty. Overall, we estimate the relative uncertainty to be about $10 \%$, or about $20 \%$ for the higher background exposures just mentioned. For the 1997 Febru-

TABLE 1

Intensities of Spectral Lines

\begin{tabular}{|c|c|c|c|c|c|c|c|c|}
\hline Date & $\begin{array}{c}\lambda \\
(\operatorname{deg})\end{array}$ & $\begin{array}{c}\delta \\
(\operatorname{deg})\end{array}$ & $\begin{array}{l}\text { Pointing } \\
\qquad\left(R_{\odot}\right)\end{array}$ & $\begin{array}{l}\text { P.A. } \\
\text { (deg east of solar north) }\end{array}$ & $\begin{array}{l}\text { Time } \\
\text { (hr) }\end{array}$ & $\begin{array}{l}\text { He I } \\
(\mathrm{R})\end{array}$ & $\begin{array}{l}\operatorname{Ly} \beta \\
(\mathrm{R})\end{array}$ & $\begin{array}{l}\text { C III } \\
(\mathrm{R})\end{array}$ \\
\hline 1996 Dec $5 \ldots \ldots \ldots \ldots$ & 74.0 & $0.05 \mathrm{~S}$ & 9.0 & 180 & 6.0 & 49.3 & 1.3 & 0.7 \\
\hline 1996 Dec $6 \ldots \ldots \ldots \ldots$ & 75.2 & $0.05 \mathrm{~S}$ & 9.0 & 180 & 6.0 & 45.8 & 1.4 & 0.4 \\
\hline 1996 Dec $7 \ldots \ldots \ldots \ldots$ & 76.1 & $0.05 \mathrm{~S}$ & 9.0 & 180 & 8.0 & 41.0 & 1.5 & 0.4 \\
\hline 1996 Dec $23 \ldots \ldots \ldots$ & 92.4 & $0.04 \mathrm{~S}$ & 9.0 & 180 & 4.0 & 21.3 & 1.3 & 0.7 \\
\hline 1997 Feb $18 \ldots \ldots \ldots$ & 150.4 & $0.04 \mathrm{~N}$ & 9.0 & 180 & 9.0 & 5.6 & 1.1 & 0.4 \\
\hline 1997 Jun 5 ........... & 255.3 & $0.06 \mathrm{~S}$ & 7.5 & 180 & 9.0 & 39.6 & 0.6 & 0.4 \\
\hline 1997 Jun $13^{\mathrm{a}} \ldots \ldots .$. & 263.1 & $0.05 \mathrm{~S}$ & 6.4 & 150 & 4.0 & 26.7 & 0.7 & 0.7 \\
\hline 1997 Aug $27^{b} \ldots \ldots$. & 334.7 & $0.04 \mathrm{~N}$ & $6.8-5.5$ & 85 & 8.0 & 7.4 & 5.4 & 2.6 \\
\hline 1997 Dec $2 \ldots \ldots \ldots \ldots$ & 70.7 & $0.05 \mathrm{~S}$ & 9.0 & 180 & 5.0 & 26.5 & 1.3 & 0.7 \\
\hline 1997 Dec $2 \ldots \ldots \ldots \ldots$ & 70.9 & $0.05 \mathrm{~S}$ & 7.0 & 180 & 4.0 & 22.6 & 1.1 & 0.5 \\
\hline 1997 Dec $4 \ldots \ldots \ldots \ldots$ & 72.7 & $0.05 \mathrm{~S}$ & 9.0 & 180 & 3.0 & 24.3 & 1.4 & 0.2 \\
\hline 1997 Dec $4 \ldots \ldots \ldots . . .$. & 72.9 & $0.05 \mathrm{~S}$ & 7.0 & 180 & 3.0 & 20.7 & 1.5 & 0.4 \\
\hline 1997 Dec 4 ............ & 73.0 & $0.05 \mathrm{~S}$ & 5.0 & 180 & 4.0 & 20.1 & 1.5 & 0.8 \\
\hline 1997 Dec $6 \ldots \ldots \ldots \ldots$ & 74.8 & $0.05 \mathrm{~S}$ & 9.0 & 180 & 3.0 & 25.2 & 1.8 & 0.3 \\
\hline 1997 Dec $6 \ldots \ldots \ldots \ldots$ & 74.9 & $0.05 \mathrm{~S}$ & 7.0 & 180 & 3.0 & 25.2 & 2.3 & 0.5 \\
\hline 1997 Dec $6 \ldots \ldots \ldots \ldots$ & 75.0 & $0.05 \mathrm{~S}$ & 5.0 & 180 & 3.0 & 20.8 & 2.4 & 1.2 \\
\hline 1997 Dec $7 \ldots \ldots \ldots \ldots$ & 75.7 & $0.05 \mathrm{~S}$ & 9.0 & 180 & 3.0 & 21.2 & 1.3 & 0.4 \\
\hline 1997 Dec $7 \ldots \ldots \ldots \ldots$ & 75.9 & $0.05 \mathrm{~S}$ & 7.0 & 180 & 3.0 & 20.4 & 1.6 & 0.5 \\
\hline 1997 Dec $7 \ldots \ldots \ldots . .$. & 76.0 & $0.05 \mathrm{~S}$ & 5.0 & 180 & 3.0 & 25.0 & 1.5 & 1.8 \\
\hline 1997 Dec $9 . . . \ldots \ldots . . .$. & 77.7 & $0.05 \mathrm{~S}$ & 9.0 & 180 & 2.0 & 27.3 & 1.1 & 0.4 \\
\hline 1998 Jun 8 .......... & 258.0 & $0.05 \mathrm{~S}$ & 9.0 & 180 & 3.0 & 13.6 & 0.9 & 0.0 \\
\hline
\end{tabular}

NotE.-Summary of UVCS data from observations of the LISM. Intensity is in rayleighs. Heliocentric longitude and latitude are computed from orbit files using the solar ecliptic coordinate system.

a Attempted observation of the Crab Nebula.

$\mathrm{b}$ Observation of $\rho$ Leo affected by emission from coronal streamer. 


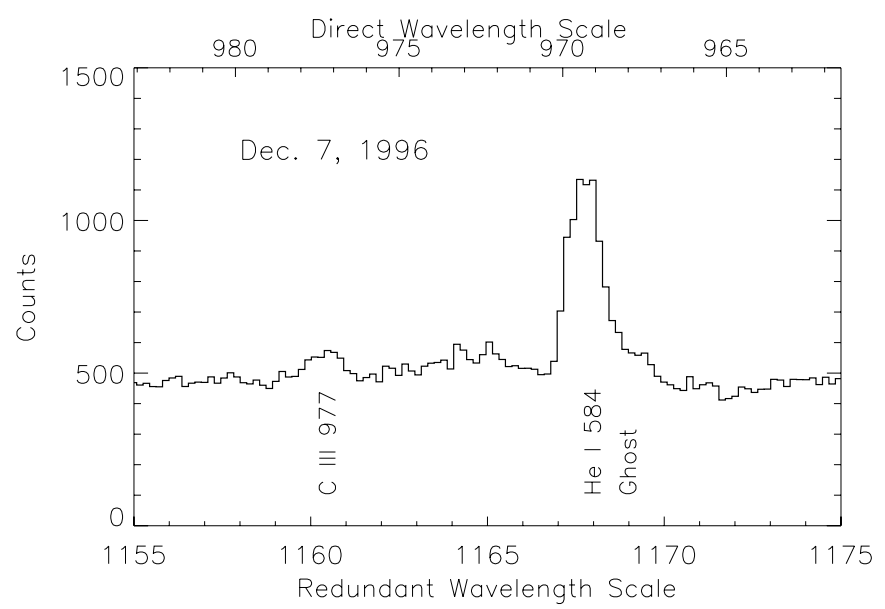

FIG. $1 a$

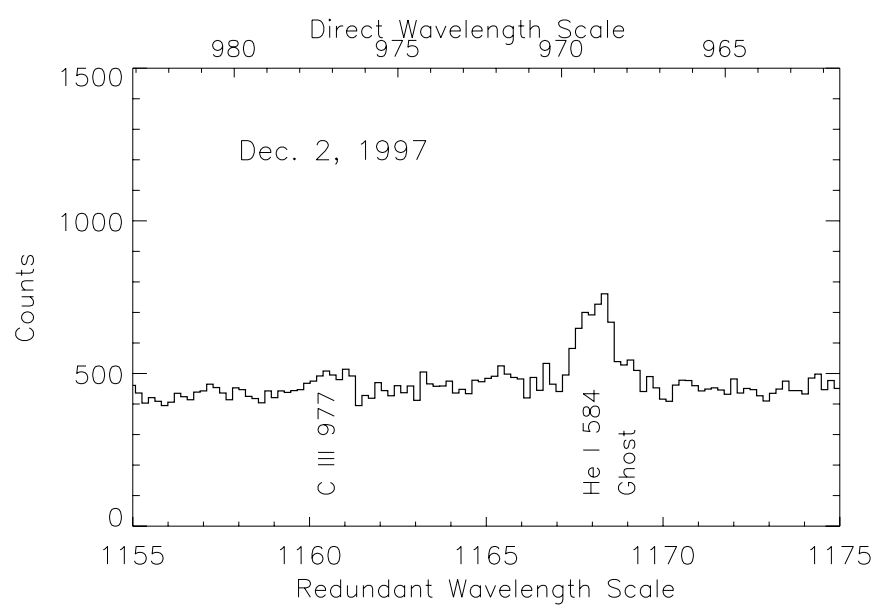

FIG. $1 b$

FIG. 1.- (a) $\mathrm{SOHO} / \mathrm{UVCS}$ spectrum at $9 R_{\text {}}$ taken on 1996 December 7 . The lower axis shows the wavelength scale for light reflected by the redundant mirror, while the upper axis shows the direct illumination wavelength scale. (b) Similar spectrum to that in (a), but obtained on 1997 December 2. While the levels of the background, the C III stray light, and the ghost are similar to those in 1996, the He I line is fainter.

ary and August observations away from the focusing cone, the very low fluxes increase the uncertainty to about $50 \%$. The sensitivity is considerably higher for $\operatorname{Ly} \beta$ and the background tends to be lower, so the uncertainty on any measurement is typically $0.3 \mathrm{R}$. However, we cannot reliably separate the interplanetary $\operatorname{Ly} \beta$ from stray light and F-corona contributions.

\section{PREDICTIONS OF A CLASSICAL MODEL OF HELIUM GLOW FOR UVCS OBSERVATIONS}

The model used in this paper computes the emission rate (in rayleighs) of the $584 \AA$ glow resulting from resonance scattering of interstellar $\mathrm{He}$ atoms illuminated by the solar $584 \AA$ emission in the optically thin regime. It is an extension of the model produced for the interpretation of $584 \AA$ intensity measurements with Prognoz 6 (Dalaudier et al. 1984). While lines of sight for Prognoz 6 probed solar distances greater than $1 \mathrm{AU}$, here we consider lines of sight approaching very near the Sun, where the focusing cone is expected to give its maximum brightness. The parameters of the model include the description of the interstellar flow of He atoms. The velocity vector $V_{w}$ is taken with $V_{w}=26 \mathrm{~km} \mathrm{~s}^{-1}$, and the ecliptic coordinates are taken as $l_{\mathrm{ecl}}=74^{\circ}, b_{\mathrm{ecl}}=-6^{\circ}$. These are the coordinates of the flow of $\mathrm{He}$ with respect to the Sun and also the axis of the focusing cone of $\mathrm{He}$ found in the downwind direction. It is obviously opposite to the velocity vector of the Sun with respect to the LIC. The helium kinetic temperature was taken as $T=6500 \mathrm{~K}$. The values of $V_{w}$ and $T$ taken here are derived both from the in situ measurements of $\mathrm{He}$ atoms on board Ulysses (Witte et al. 1996) and from the ground-based determination of the LIC motion from Doppler triangulation of interstellar absorption lines (Lallement \& Bertin 1992). They coincide because the flow of interstellar $\mathrm{He}$ atoms is not modified at the heliopause interface, at variance with the case of $\mathrm{H}$. The density of $\mathrm{He}$ at infinity (say, at solar distance greater than $20 \mathrm{AU}$ ) was taken as $n_{0}(\mathrm{He})=0.015 \mathrm{~cm}^{-3}$ (Witte et al. 1996; Gloeckler \& Geiss 1998). Since we are in the optically thin regime, the value of $n_{0}(\mathrm{He})$ is a multiplicative factor for the $584 \AA$ pattern. These parameters are compatible with values derived from pick-up ion data and more recently from $E U V E$ observations of the $584 \AA$ glow using the geocoronal helium as an absorbing cell (Flynn et al. 1998).

Interstellar He atoms suffer losses from ionization along their trajectory. Solar photoionization is the major contributor at distances more than $0.3 \mathrm{AU}$, and it scales as $r^{-2}$. Therefore, it can be integrated analytically along the Keplerian orbit of the He atom and is prescribed by the photoionization rate at $1 \mathrm{AU}$, of the order of $\beta_{\text {ion }}=10^{-7} \mathrm{~s}^{-1}$, as previously estimated from solar EUV flux (Rucinski et al. 1996). Fortunately, the Charge, Element, and Isotope Analysis System (CELIAS) instrument aboard $\mathrm{SOHO}$ (Hovestadt et al. 1995) includes one solar EUV channel Solar EUV Monitor (SEM) that monitors the solar flux at $304 \AA$ (the main solar radiation that photoionizes $\mathrm{He}$ atoms). The radiometric calibration has been compared with results from sounding rocket flights (Judge, McMullin, \& Ogawa 1999). From these solar measurements the value of $\beta_{\text {ion }}$ was estimated as a function of time and provided to us for the present analysis (D. McMullin 2001, private communication). As indicated in Table 2, it increased from $0.65 \times 10^{-7} \mathrm{~s}^{-1}$ in 1996 December to $0.85 \times 10^{-7} \mathrm{~s}^{-1}$ in 1997 December.

Below $r=0.3$ AU, electron impact ionization by hot solar wind electrons may become important (Holzer 1977). However, it does not scale as $r^{-2}$ because of the electron temperature dependence of the ionization rate and decrease of solar wind electron temperature with increasing $r$. As a consequence, the ionization rate can no longer be integrated analytically along the trajectory.

In the limited scope of this paper, where UVCS He glow data are presented and discussed, we have used the analytical $r^{-2}$ description of the ionization but we have left free the parameter $\beta_{\text {ion }}$ in order to include (at least partially) the effect of electron impact ionization. This is the same method as the one employed by Witte et al. (1996) to fit the in situ measurements of He-density data of the GAS experiment on Ulysses. Doing so, Witte et al. found that for indirect orbits of the atoms, i.e., atoms that have gone through perihelion, a stronger ionization rate is inferred, showing that electron impact has a stronger influence on these atoms than on atoms on direct orbits (that have not yet passed through perihelion). The analysis by Witte et al. estimates the extinc- 
TABLE 2

Comparison of Observed and Model Fluxes

\begin{tabular}{|c|c|c|c|}
\hline Parameter & 1996 December & 1997 December & 1998 June \\
\hline UVCS measured intensity $(\mathrm{R})$................. & 45 & 23 & 13.6 \\
\hline SUMER normalized 584 A solar flux ........................... & 1.00 & 1.20 & 1.20 \\
\hline 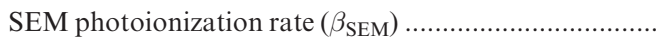 & $0.65 \times 10^{-7}$ & $0.85 \times 10^{-7}$ & $1.00 \times 10^{-7}$ \\
\hline 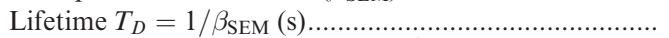 & $1.54 \times 10^{7}$ & $1.17 \times 10^{7}$ & $1.00 \times 10^{7}$ \\
\hline Model intensity $\times$ normalized $584 \AA$. & 94 & 66 & 46 \\
\hline Required ionization rate $\beta_{\text {req }} \ldots \ldots \ldots \ldots . . .$. & $0.94 \times 10^{-7}$ & $1.31 \times 10^{-7}$ & $1.56 \times 10^{-7}$ \\
\hline 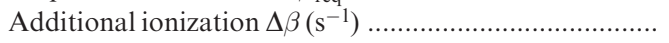 & $0.29 \times 10^{-7}$ & $0.46 \times 10^{-7}$ & $0.56 \times 10^{-7}$ \\
\hline 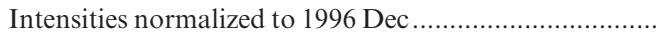 & 94 & 40 & 24 \\
\hline Required ionization rate $\beta_{\text {req }}$ (with normalization) .......... & $0.65 \times 10^{-7}$ & $0.98 \times 10^{-7}$ & $1.22 \times 10^{-7}$ \\
\hline Additional ionization $\Delta \beta\left(\mathrm{s}^{-1}\right)$ (with normalization) ...... & 0 & $0.14 \times 10^{-7}$ & $0.22 \times 10^{-7}$ \\
\hline
\end{tabular}

tion factor for electron impact ionization inside 1.3 AU to be 0.22 , implying twice the value of the photoionization extinction.

The model distribution (which has a cylindrical symmetry around the wind axis) of $\mathrm{He}$ atoms in the vicinity of the Sun is represented in Figure $2 a$ in a plane containing the wind axis. In this case, the $\mathrm{He}$ density was normalized to the $\mathrm{He}$ density at infinity, $n_{0}$. Two effects are conspicuous in this figure. The He density distribution is flat and uniform except in two areas. One area is in front of the Sun, where a cavity is formed by ionization in the incoming flow. The second area is downwind along the wind axis, where the density is very much enhanced by gravitational focusing (all trajectories are hyperbolae that cross the downwind semiaxis). Both the size of the ionization cavity and the maximum enhancement factor will depend strongly on the ionization rate. In this particular case, the lifetime versus ionization was taken to be $T_{D}=0.8 \times 10^{7} \mathrm{~s}$, corresponding to $\beta_{\text {ion }}=1.25 \times 10^{-7}$ $\mathrm{s}^{-1}$. Figure $2 b$ is a contour plot of the same relative density model for a better estimate of the density-enhancement factor in the focusing cone and the depletion factor in the ionization cavity near the Sun. For this particular case, the interstellar helium temperature and velocity were taken to be $T=7000 \mathrm{~K}$ and $25.4 \mathrm{~km} \mathrm{~s}^{-1}$. These slight differences from our nominal case $\left(T=6500 \mathrm{~K}, V=26 \mathrm{~km} \mathrm{~s}^{-1}\right)$ do not significantly change the overall pattern.

From the same model parameters, the He $584 \AA$ emissivity is plotted in Figure 3 for a region close to the Sun in the form of isocontours. Numbers labeling the isocontours are in photons $\mathrm{m}^{-3} \mathrm{~s}$, multiplied by 1000 . With respect to the density distribution of Figure $2 a$, the $r^{-2}$ dependence of the solar excitation factor changes the shape of the distribution (emissivity versus density). Computation of the local excitation rate must take into account the line profile of the solar He I $584 \AA$ (disk averaged) because the velocity of He atoms, in their free fall to the Sun, may be comparable to the solar line width (Doppler dimming). The excitation is described by its value at line center and at $1 \mathrm{AU}, g_{0}=0.58 \times 10^{-5} \mathrm{~s}^{-1}$, while the solar line width was taken as $0.081 \AA$ (half-width at $1 / e$ ), or $42 \mathrm{~km} \mathrm{~s}^{-1}$. These parameters are derived from the Solar Ultraviolet Measurement of Emitted Radiation (SUMER) instrument measurements (K. Wilhelm 2001, private communication). Note that both the solar flux at line center $\left[7 \times 10^{9}\right.$ photons $\left.\left(\mathrm{cm}^{2} \mathrm{~s} \AA\right)^{-1}\right]$ and line width from SUMER are significantly smaller than the previous estimates by Phillips, Judge, \& Carlson (1982) and Ogawa, Phillips, \& Judge (1997) $\left(g_{0}=1.62 \times 10^{-5} \mathrm{~s}^{-1}\right.$ and $\left.57 \mathrm{~km} \mathrm{~s}^{-1}\right)$. The SUMER results were obtained in 1996 June and are more relevant to the UVCS observations than the older observations. Accordingly, we adopt them in the following. Note the pear shape of the isocontours, with the elongation along the downwind semiaxis.

The intensity (or emission rate ) in rayleighs is computed by integrating the volume emissivity $\epsilon(\ell)$ (photons $\mathrm{cm}^{-3} \mathrm{~s}^{-1}$ ) along the line of sight:

$$
I(R)=10^{-6} \int \epsilon(\ell) d \ell .
$$

The phase function for He resonance scattering is taken into account. It can be remarked that $g_{0}$, as well as $n_{0}$ and the UVCS calibration factor, are all scaling factors playing a similar role in the fitting process. Therefore, only one parameter of the model was kept free to fit the model He I intensities to UVCS data: the $r^{-2}$ dependent photoionization rate $\beta_{\text {ion }}$ (with the lifetime $T_{D}$ of one $\mathrm{He}$ atom at $1 \mathrm{AU}=1 / \beta_{\text {ion }}$, of the order of $10^{7} \mathrm{~s}$ or 115 days, it is clear that ionization of $\mathrm{He}$ is significant only well within $1 \mathrm{AU}$ ).

To give an idea of what the focusing cone should look like in $584 \AA$ light, the model isocontours of the He I brightness (rayleighs) are represented in Figure 4 for an observer on the $+Z$ ecliptic axis at a solar distance of $3 \mathrm{AU}$. The pear shape of emissivity isocontours is still preserved when one goes to this particular geometry of observation. On the contrary, Figure 5 shows the model isocontours of the $\mathrm{He}$ I interplanetary emission rate for an observer located at $1 \mathrm{AU}$ downwind (ecliptic longitude $74^{\circ}$ ), relevant to $\mathrm{SOHO} /$ UVCS observations around December 5 of each year. The ionization lifetime was taken as $0.91 \times 10^{7} \mathrm{~s}$ in order to reproduce the observed average level of intensity. The points indicate the positions of UVCS measurements at 5, 7, and $9 R_{\odot}$, with position angle (P.A.) $=180^{\circ}$. The slight offset between solar axis rotation and ecliptic axis was ignored. Directions of UVCS are located south of the Sun (negative latitudes). The isophotes are round for this particular geometry and symmetrical about a plane. The position of the observer and the helium cone axis will always define a symmetry plane for the intensity pattern, which projects along the cone axis on the plane of the sky, as shown by all maps produced for various locations of the observer. At the downwind position, the cone axis projects along the latitude axis at longitude $\lambda=254^{\circ}$. Note that the maximum intensity lies between the Sun (at $\lambda=254^{\circ}, \beta=0^{\circ}$ ) and the UVCS points of observations.

The distributions of model intensity along the latitude axis (which corresponds to P.A. $=0^{\circ}$ and P.A. $=180^{\circ}$ ) are plotted in Figure 6 for the five dates of UVCS observations 


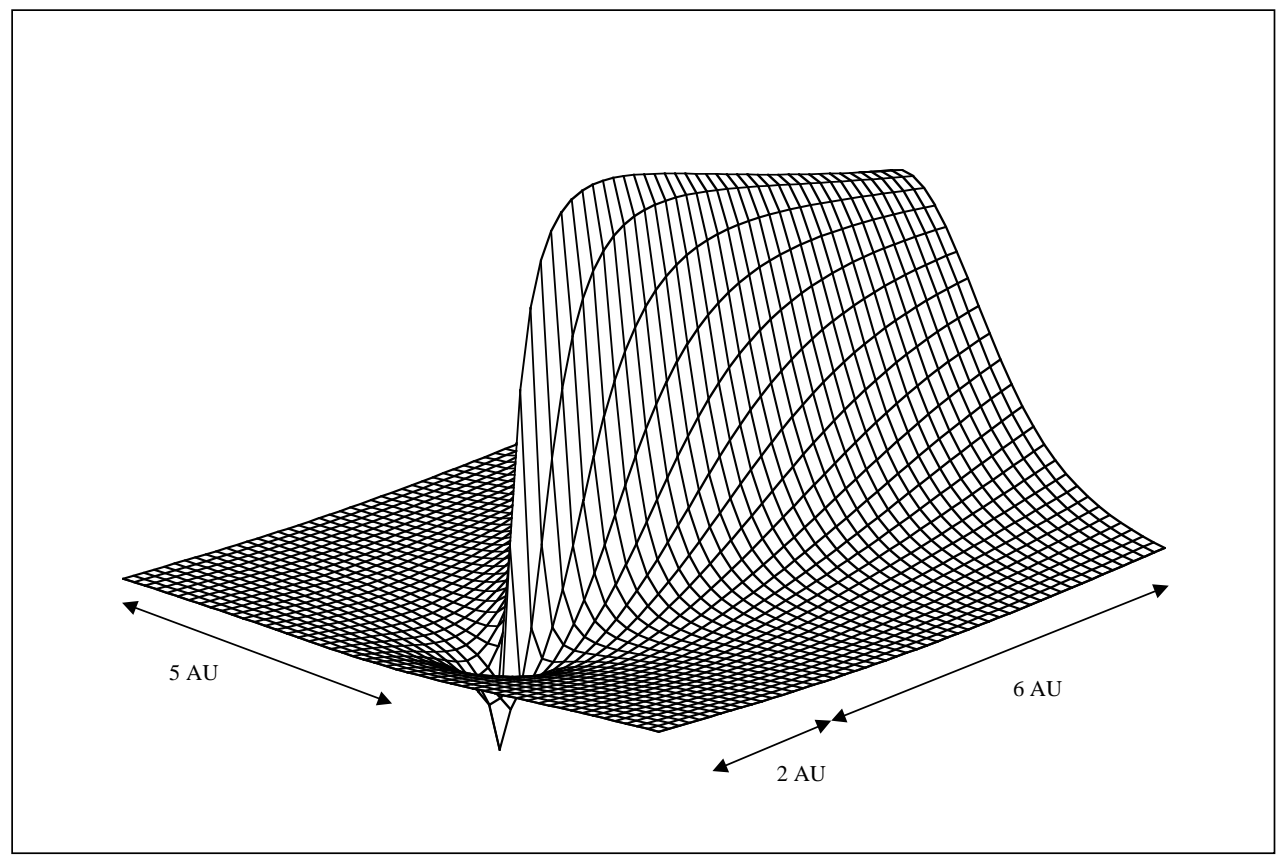

FIG. $2 a$

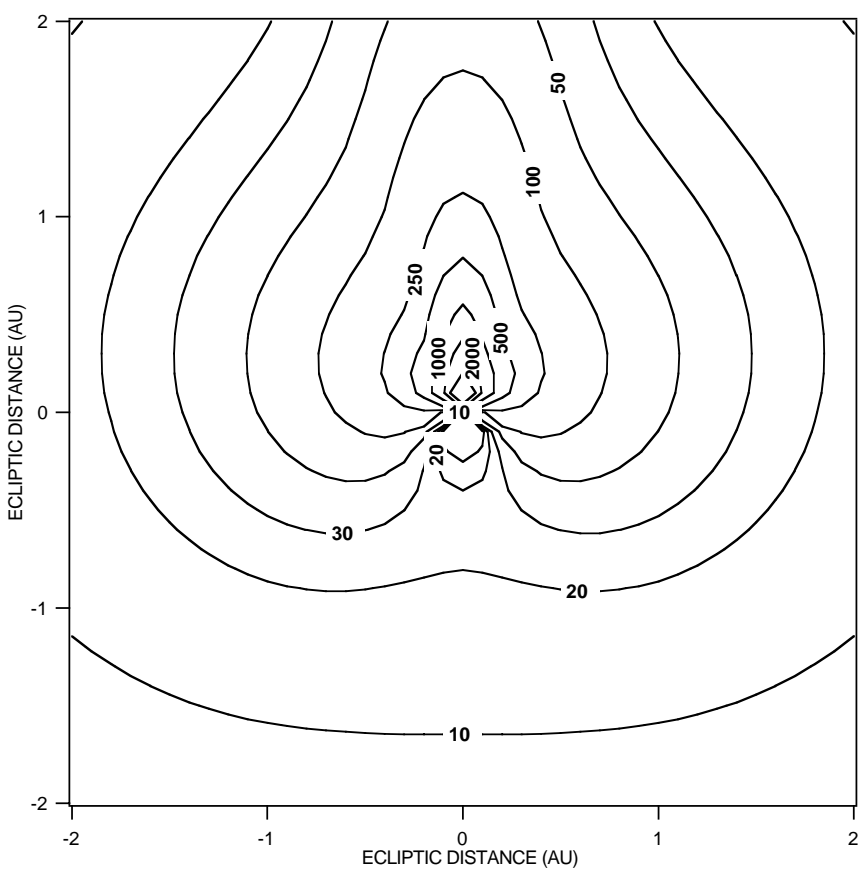

FIG. $2 b$

FIG. 2.- (a) Model of the relative distribution of He atoms of interstellar origin in the solar system, represented as a function of position in the solar system $(X$ and $Y$ in $\mathrm{AU}$ ) in a plane containing the wind axis. The distribution is axisymmetric around the wind axis. The Sun is at center of coordinates axis; along the $Z$-axis is plotted the ratio of local density to the density "at infinity" of He atoms. The so-called focusing cone, caused by the effect of solar gravitation, is elongated along the downwind axis. There is a cavity of ionization upwind from the Sun. Model parameters are $T=7000 \mathrm{~K}, V=25.4 \mathrm{~km} \mathrm{~s}{ }^{-1}$, ecliptic longitude $l=90^{\circ}$, latitude $b=0^{\circ}$, lifetime of He vs. ionization $0.8 \times 10^{7} \mathrm{~s}$ at $1 \mathrm{AU}$. (b) Same model shown as a contour plot of the ratio of local density to density at infinity.

in 1998 December (see Table 1). They are quite similar: from December 2 to $9, \mathrm{SOHO}$ moved by only $7^{\circ}$ in ecliptic longitude, and this does not affect the intensity profile very much. The abscissae are graduated in degrees of latitude, $\left(R_{\odot}=0.25\right.$ for latitude); negative values are for P.A. $=180^{\circ}$. The maximum is located at $1.5 R_{\odot}$ south.

\section{COMPARISON OF DATA TO MODEL}

To help in describing our results contained in Table 1, we have divided the observations into three groups based on the position of $\mathrm{SOHO} / \mathrm{UVCS}$ relative to the He focusing cone as determined by the heliocentric longitude of the 


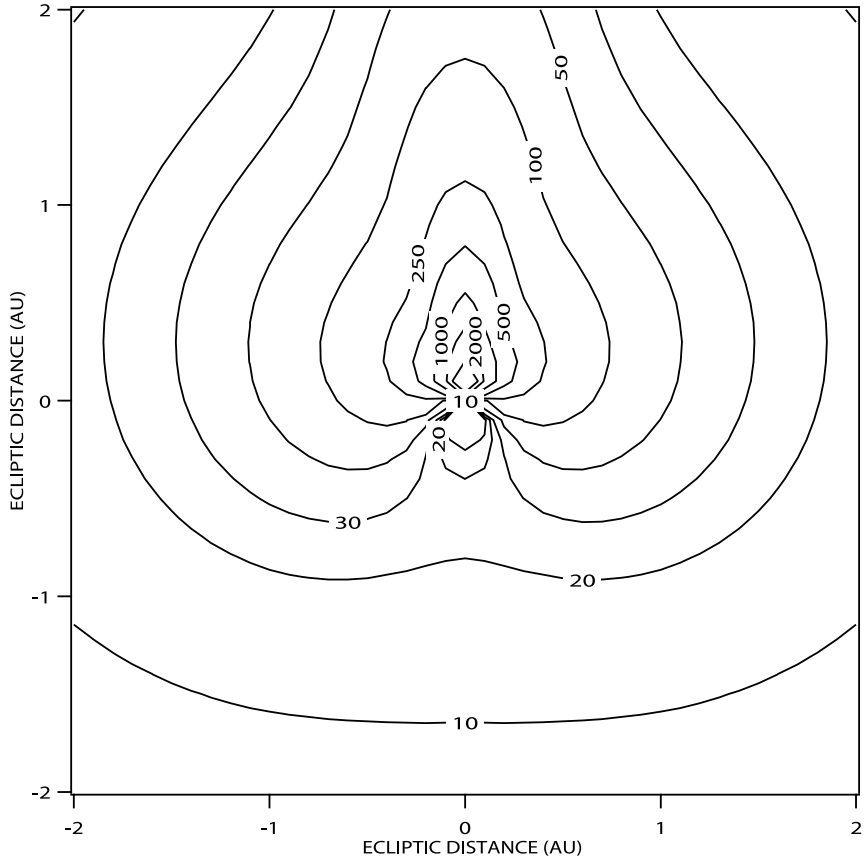

FIG. 3.- Model distribution of emissivity of He I $584 \AA$ in the solar system due to resonance scattering of solar photons by He atoms. Isoemissivity contours are plotted in the ecliptic plane, labeled in 0.001 photons $\mathrm{m}^{-3} \mathrm{~s}$. The distribution is axisymmetric around the wind axis assumed to be in the ecliptic plane. Parameters of the model are $T=6500 \mathrm{~K}, V=26 \mathrm{~km} \mathrm{~s}^{-1}$, $T_{D}=1.1 \times 10^{7} \mathrm{~s}$. The excitation factor at $1 \mathrm{AU}$ was taken as $0.58 \times 10^{-5}$ $\mathrm{s}^{-1}$, and the solar $\mathrm{He} \mathrm{I}$ line is assumed to have a Gaussian shape with $42 \mathrm{~km}$ $\mathrm{s}^{-1}$ width at $1 / e$ as measured by SUMER.

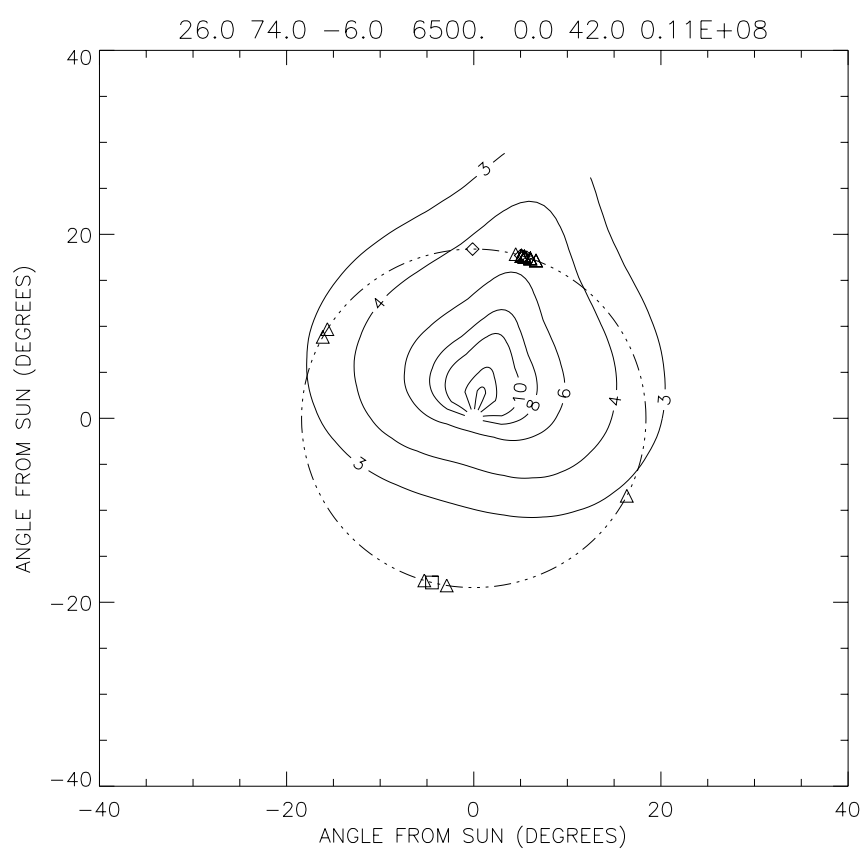

FIG. 4.-Model distribution of emission of He I $584 \AA$ An the solar system, as would be seen from a point located on the north ecliptic axis, at $3 \mathrm{AU}$ above the ecliptic plane. The emission is the integral of emissivity along each line of sight (optically thin regime) and is indicated with isophote contours in rayleighs. Parameters of the model are the same as for Fig. 3. The orbit of the Earth projected on the ecliptic plane is shown as the dot-dashed line, and the triangles show the positions of $\mathrm{SOHO}$ at the times of UVCS observations (always oriented near the solar disk). The Earth's orbit gives the linear scale of the pattern. The cluster of triangles in the upper right are the December observations, and those in the lower left are June measurements.

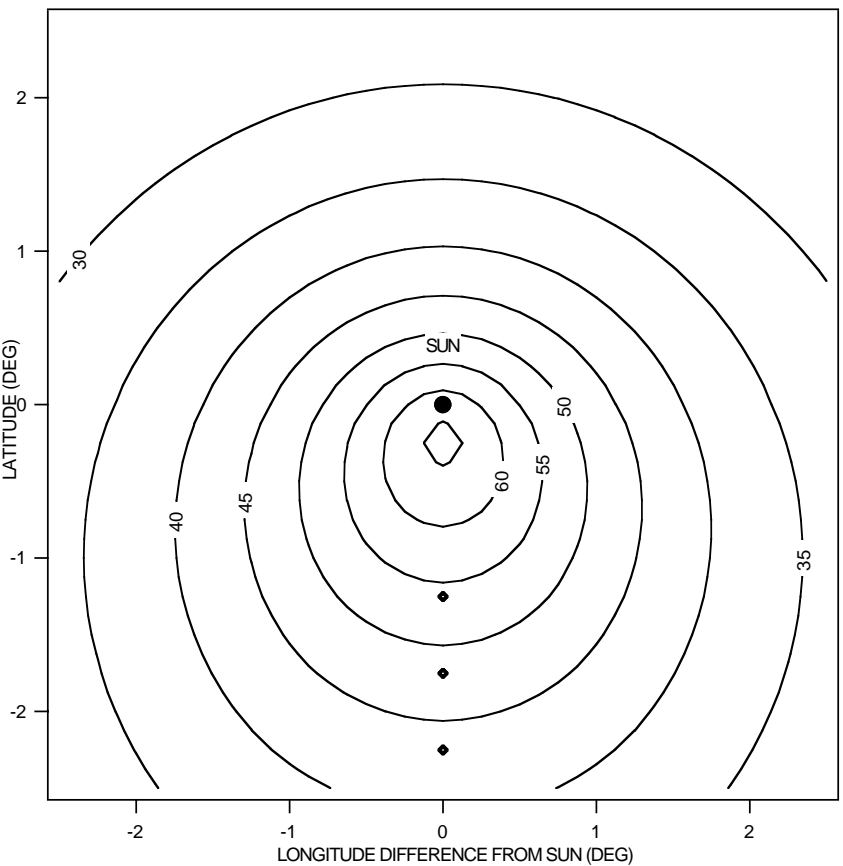

FIG. 5.-Model isophotes as seen from $\mathrm{SOHO}$ position in December at ecliptic longitude $74^{\circ}$. The FOV is graduated in ecliptic coordinates of the line of sight (deg). Diamonds represent the directions of sight of UVCS at 5, 7 , and $9 R_{\odot}$ below the Sun (southward). Parameters of the model are the same as for Fig. 3. The lifetime of He vs. ionization was chosen to fit globally the intensity observed in 1996 December at $1.1 \times 10^{7} \mathrm{~s}$ at $1 \mathrm{AU}$. The maximum intensity in the model is at about 0.3 (or $1.2 R_{\odot}$ ) below the center of the Sun. The dark circle indicates the position of the Sun.

spacecraft, $\lambda$. The first group consists of those observations when $\lambda \sim 74^{\circ}$ and the UVCS line of sight is directed through the focusing cone toward the south coronal hole. Two sets of data from 1996 December 5-7 and 1997 December 2-9 fit into this group. We estimate that relative uncertainties for the 1996 December data are $\pm 5 \mathrm{R}$. As the 1997 December observations are $3.5 \mathrm{hr}$ per heliocentric height, as compared with $6-8 \mathrm{hr}$ observations in 1996 , the uncertainty of the

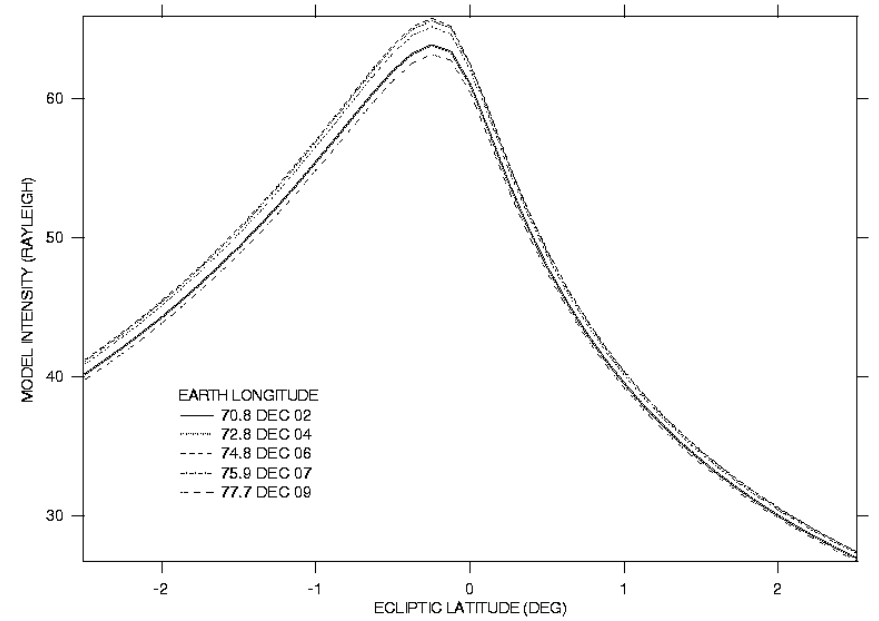

FIG. 6.-Model He I $584 \AA$ A intensity in a vertical plane (perpendicular to the ecliptic) through the center of the Sun, as a function of impact parameter (in rayleighs) of the line of sight for the 5 days of observations in 1997 December (Table 1). North is positive, at right from center. They are quite similar to each other, all showing a maximum at $1.2 R_{\odot}$ below the Sun's center. Parameters of the model are the same as for Fig. 5. 


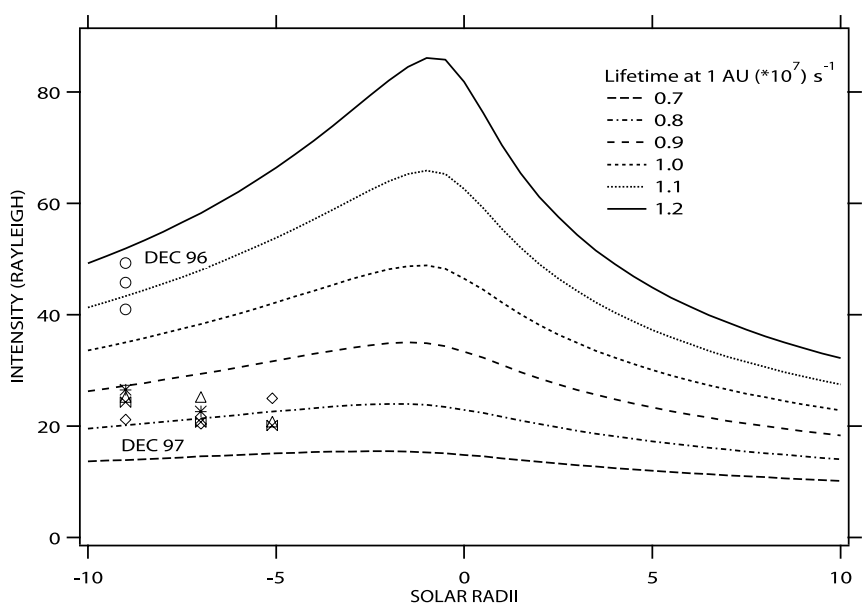

FIG. 7.-UVCS measured He I $584 \AA$ intensities (rayleighs) at $9 R_{\odot}$ south of the Sun in 1996 December and at 5, 7, and $9 R_{\odot}$ south of the Sun in 1997 December, compared with six model curves differing in lifetime: $0.70,0.80$, $0.90,1.00,1.10$, and $1.20 \times 10^{7} \mathrm{~s}$ at $1 \mathrm{AU}$. The most intense model curve corresponds to the largest lifetime (smallest ionization rate).

1997 observations is increased. Contamination of $\mathrm{He}$ I intensities by F-corona or instrumental stray light appears to be negligible, as the $\mathrm{C}$ III intensity is less than $1 \mathrm{R}$. Based on the ratio of $\operatorname{Ly} \beta$ and $C$ III disk intensities, about half of the much weaker Ly $\beta$ intensity is probably caused by instrument or F-corona contamination.

The second group consists of those observations taken when $\lambda \sim 254^{\circ}$, which is aligned with the velocity vector of the interstellar wind. The UVCS line of sight intersects the focusing cone as it angles below the ecliptic plane on the far side of the Sun. Three sets of data from 1997 June 5 and 13 and 1998 June 8 are included in this group. Finally, the remaining data taken on 1997 February 18 and 1997 August 27 are grouped together as observations where the UVCS line of sight is neither parallel nor antiparallel to the focusing cone.

In Figure 7, we compare the 1996 and 1997 December data points with model curves, computed for longitude $74^{\circ}$, representative of the whole period of a few days around passage to downwind position. The size model curves differ only by the value of ionization: $T_{D}=0.80,0.90,1.00,1.10$, and $1.20 \times 10^{7} \mathrm{~s}$, which encompass the UVCS measurement points. This shows the enormous sensitivity of the He I intensity to the ionization because, in this particular geometry, UVCS observes atoms that have passed very near the Sun and have suffered a significant ionization loss. A value of $1.06 \times 10^{7} \mathrm{~s}$, or $\beta_{\text {ion }}=0.94 \times 10^{-7} \mathrm{~s}^{-1}$, provides a fit to the intensities in 1996 December, while a shorter lifetime, about $0.82 \times 10^{7} \mathrm{~s}$, fits the average of the observed values at 5, 7, and $9 R_{\odot}$ in 1997 December. The slight predicted increase in intensity with decreasing distance from the Sun is not seen in the data, but the uncertainties are larger than the predicted trend.

Now, comparison of the two December data sets from the first group reveals that the He focusing cone intensity falls 50\% from an average of $45 \mathrm{R}$ in 1996 to $23 \mathrm{R}$ in 1997. This is certainly the result of the variation of ionization rate of He atoms over 1 yr. Indeed, as illustrated in Figure 8, the model intensity seen from the downwind position (December) at $7 R_{\odot}$ south of the Sun is a very strongly decreasing function of the ionization rate (assumed here to have $1 / r^{2}$ dependence). Relevant data for 1996 and 1997 December

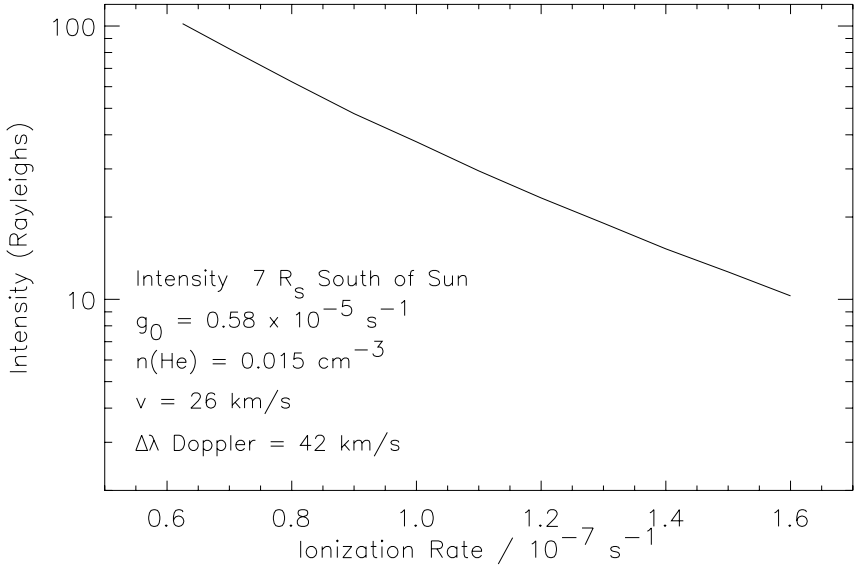

FIG. 8.-Model intensity (rayleighs) at $7 R_{\odot}$ south of the Sun seen from downwind position (around December 6) as a function of the ionization rate, showing the strong dependence over the possible range of the photoionization rate experienced over the early rise of the solar cycle.

are summarized in Table 2. Let us assume first that the calibration of UVCS is exact, along with the values of $n_{0}$ (helium density at infinity) and excitation factor $g_{0}$ obtained from SUMER. For 1996 December, the SEM/CELIAS data measured a photoionization rate $\beta_{\mathrm{SEM}}=0.65 \times 10^{-7} \mathrm{~s}^{-1}$, or $T_{D}=1.54 \times 10^{7} \mathrm{~s}$. The He I model intensity would amount to $94 \mathrm{R}$, while the measured value is $45 \mathrm{R}$. This implies that the ionization rate is larger than the measured photoionization rate. Using the curve in Figure 8 , the value $\beta_{\text {req }}$ required to fit $45 \mathrm{R}$ is $0.94 \times 10^{-7} \mathrm{~s}^{-1}$, larger than $\beta_{\text {SEM }}$ by $\Delta \beta=0.29 \times 10^{-7} \mathrm{~s}^{-1}$. We attribute this additional ionization to electron impact ionization at the time of solar minimum (1996 December).

One year later, with the increase of solar activity, $\beta_{\mathrm{SEM}}$ had increased to $0.85 \times 10^{-7} \mathrm{~s}^{-1}$, but the solar He I line flux had also increased by $20 \%$ (SUMER; K. Wilhelm 2001, private communication). With these parameters the model predicts $66 \mathrm{R}$ instead of the observed value of $23 \mathrm{R}$. This intensity deficit implies $\beta_{\text {req }}=1.31 \times 10^{-7} \mathrm{~s}^{-1}$, or an additional ionization rate of $\Delta \beta=0.46 \times 10^{-7} \mathrm{~s}^{-1}$, significantly more than a year previously, that we attribute again to electron impact ionization.

Since there is some uncertainty in the UVCS calibration and the interstellar helium parameters, we also consider the extreme case where these uncertainties conspire to match the measured intensity in 1996 December with the total ionization rate $\beta_{\text {req }}=\beta_{\mathrm{SEM}}=0.65 \times 10^{-7} \mathrm{~s}^{-1}$. Applying the same factors to the 1997 December data and taking the $20 \%$ increase in He I illuminating flux from the Sun into account, one would expect $40 \mathrm{R}$. From Figure 8, $\beta_{\text {req }}=0.99 \times 10^{-7} \mathrm{~s}^{-1}$, larger than $\beta_{\mathrm{SEM}}=0.85 \times 10^{-7} \mathrm{~s}^{-1}$ by $\Delta \beta=0.14 \times 10^{-7} \mathrm{~s}^{-1}$.

In conclusion, comparison of the 1996/1997 December data shows that more ionization is needed than is provided by EUV photoionization alone and that this additional ionization increased significantly over 1 yr by about $\Delta \beta=0.14 \times 10^{-7} \mathrm{~s}^{-1}$.

The second group, consisting of the June observations, also demonstrates a reduction in $\mathrm{He} \mathrm{I}$ intensity in the cone farther from the Sun and confirms a decrease in neutral helium density as solar activity increased from 1997 to 1998. The 1997 June 5 recorded He I intensity of $40 \mathrm{R}$ is $10 \%$ lower than the values 6 months earlier. With the same instrument 


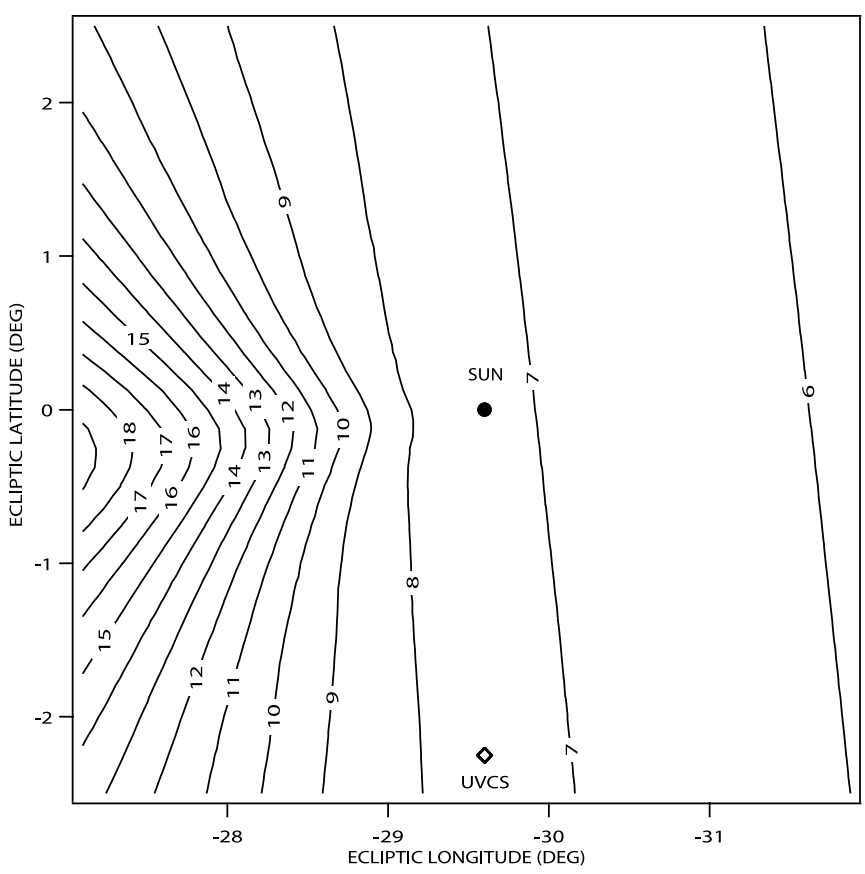

FIG. 9.-Model isophotes as seen from $\mathrm{SOHO}$ position in 1997 February, almost sidewind. The Sun is at $-29^{\circ} .6$ ecliptic longitude and $0^{\circ}$ latitude. Parameters of the model are as for previous figures, with a lifetime of $1.1 \times 10^{-7} \mathrm{~s}$. The diamond indicates the position of UVCS pointing, $9 R$ south of the Sun. The model predicts an intensity of $7.5 \mathrm{R}$, while the measured intensity was $5.6 \mathrm{R}$.

configuration, this value drops to $13 \mathrm{R}$ for 1998 June 6 , a decrease by more than a factor of 2, i.e., a decrease of the same order as for the December observations. On the other hand, $\mathrm{Ly} \beta$ fluxes seem to be generally higher in the upwind (June) than in the downwind (December) direction, although the uncertainty is large.

Finally, the third group of observations gathers data from when the UVCS line of sight deviates substantially from the direction of the interstellar wind. A map of model isophotes for ecliptic longitude near $152^{\circ}$ is represented in Figure 9, with the location of the UVCS observation (diamond) on 1997 February 18. Model parameters are the same as for Figure 5, adjusted for the 1996 December measurements. At 5.6 R, the measured intensity is smaller than the model intensity of $7.6 \mathrm{R}$, already suggesting a decline in the helium density. He I intensities for the final observation on 1997 August 27 were extracted from observations of the star $\rho$ Leo from sections of the spectrograph slit far from the stellar spectrum: $\lambda=337^{\circ}$, and the UVCS slit is on the solar east limb as opposed to the south coronal hole. The UVCS line of sight does not intersect the focusing cone, yet a 7.4 R $\mathrm{He} \mathrm{I}$ intensity is observed along with an intensity of $5.4 \mathrm{R}$ for $\operatorname{Ly} \beta$. With the UVCS slit astride the solar streamer belt, it is likely that much of the strong $\operatorname{Ly} \beta$ comes from background coronal light, which is an order of magnitude brighter than at the poles. It is difficult to dismiss the $\mathrm{He}$ I intensity as an artifact, but the uncertainty is larger.

An overview of UVCS intensity data and comparison with the model is given in Figure 10. The intensities from UVCS plotted as a function of time show a general decrease with time. In the bottom panel, all intensities were divided by the model intensity (with the standard parameters used in Fig. 5), and here the trend is even clearer: the He density in the focusing cone near the Sun has decreased during the
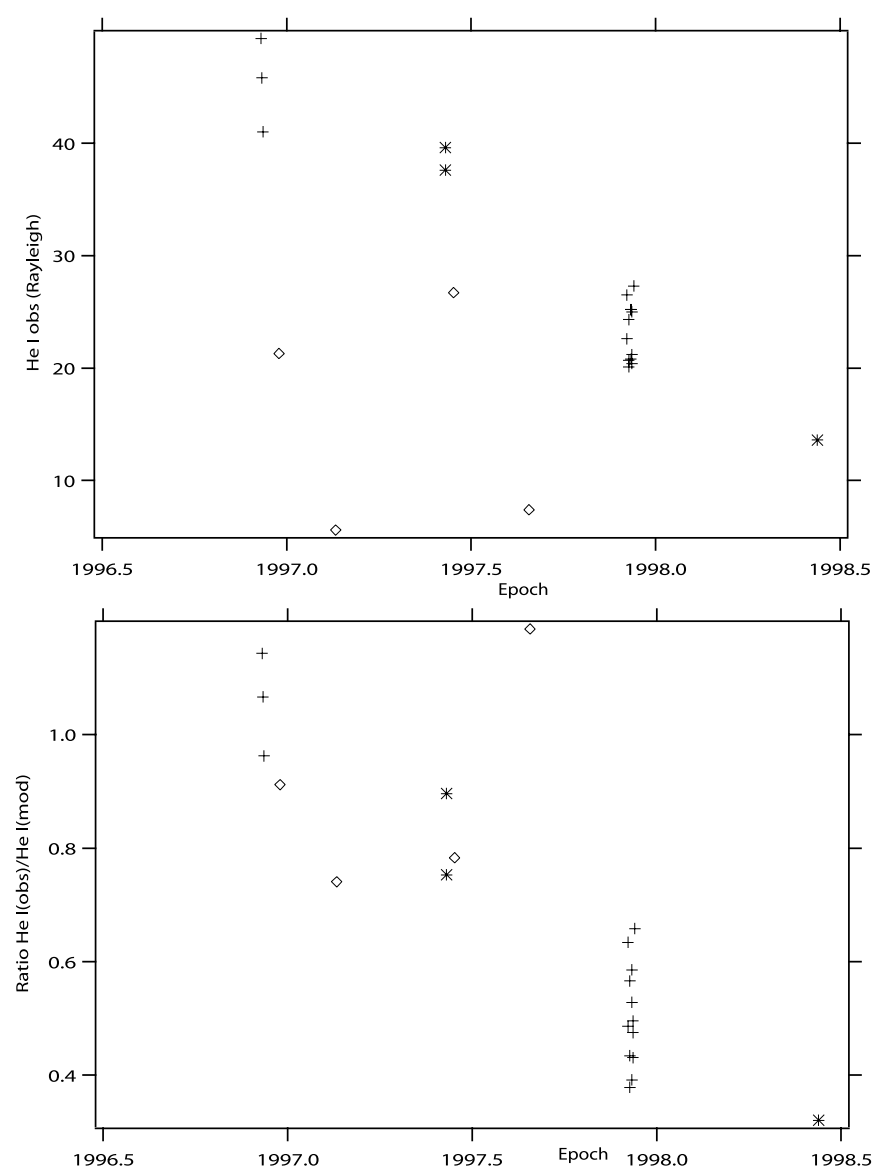

FIG. 10.-Plots of He I intensity (top) and observed intensity (bottom) divided by predicted intensity with a lifetime of $1.1 \times 10^{7} \mathrm{~s}$ as a function of observation date. The symbols indicate observations made near the axis of the focusing cone in December ( plus signs) and June (asterisks). Diamonds indicate observations obtained more than $6^{\circ}$ from the axis. The decrease is the result of an increase in ionization rate with the approach to solar maximum. The inferred level of increase is greater than that caused by EUV photoionization alone and results from an increase in solar wind electron impact ionization.

ascending phase of the solar cycle. The decrease is about a factor of 3. Since the intensity of the He I $\lambda 584$ line from the solar disk has increased, the He density decrease is even larger. Still, let us assume that the solar $\lambda 584$ flux was constant. In spite of the scatter in the data, the trend noted above from 1996 December to 1997 December is confirmed and, in particular, continues to 1998 June, where the difference between the observations and the model with constant $\beta_{\text {ion }}$ is more than a factor of 3. Applying to this 1998 June point the same analysis (Table 2) as for 1996 and 1997 December, it is found that the required ionization rate is $1.56 \times 10^{-7} \mathrm{~s}^{-1}$, compared with $\beta_{\mathrm{SEM}}=1.00 \times 10^{-7} \mathrm{~s}^{-1}$, and requires a substantial additional $0.56 \times 10^{-7} \mathrm{~s}^{-1}$ ionization rate. In the extreme case that calibration uncertainties and interstellar helium parameter uncertainties conspired to match the SEM ionization rate in 1996 December, an additional rate of $0.22 \times 10^{-7} \mathrm{~s}^{-1}$ would still be needed, continuing the earlier trend. Although a part of the decrease observed by UVCS must be attributed to the increase of solar photoionization, a large fraction of it is not accounted for. We interpret the additional decrease as the variation of the effect of electron impact ionization in the solar wind, increasing toward solar maximum. 


\section{DISCUSSION}

The comparison of the different sets of UVCS data with the classical model of the helium flow shows very clearly that a source of ionization acting close to the Sun is missing in the model and suggests that this ionizing agent is highly variable with time. Electron impact ionization is very likely responsible for the He ionization, becoming the dominant effect within a fraction of an AU from the Sun, in agreement with predictions (Rucinski \& Fahr 1989) and the most recent Ulysses results (Witte et al. 1996). Simultaneously, such observations are shown to be a powerful tool to study the collisional ionization by the electrons and the relative contributions of photoionization and collisional processes close to the Sun. The diagnostics are the intensity level, the pattern itself (in particular the distance to the Sun of the location of the maximum emission), and the temporal variability. Temporal variability is detectable possibly even at a daily scale, as suggested by the December data of group 1 . Some of this variability results from changes in the solar 584 A illuminating flux. The solar Ly $\alpha$ flux shows a 10\% rotational modulation near solar minimum, increasing to $20 \%$ near solar maximum (Woods et al. 2000). The He i 584 amplitude should be at least as large. Some of the variability may also arise from changes in the solar wind density and temperature. The solar wind, especially at low latitudes, is highly variable at any temporal scale. Because solar wind variations propagate at the wind speed, i.e., about $2 R_{S}$ $\mathrm{hr}^{-1}$, inhomogeneities of size of the order of $20-40 R_{\odot}$, (i.e., large enough to influence the integrated intensity) can appear during 10-20 hr. As a consequence, it is not surprising to observe day-to-day variations of the glow if electron impact ionization is a dominant effect in a fraction of the line of sight contributing significantly to the emission.

As a final note, Bertaux \& Blamont (1976) have suggested that if the dust is neutral, it is likely that a dust cone exists similar to that of helium. SOHO/UVCS observations reveal no obvious correlation of $\mathrm{C}$ III flux with distance from the focusing cone axis, but we do not have detailed predictions for the scattered C III line intensity.

The observations reported here were made possible by the efforts of the $\mathrm{SOHO}$ operations team. The analysis has benefited greatly from two meetings of the Helium Focusing Cone team organized by E. Möbius at the International Space Sciences Institute. J. L. Bertaux and R. Lallement thank Jean-Claude Lebrun from Service d'Aeronomie for his help with model representation and Francis Dalaudier, who provided the code we have adapted. The UVCS observations were performed and analyzed under NASA grant NAG 5-10093 to the Smithsonian Astrophysical Observatory. $\mathrm{SOHO}$ is an international cooperative mission operated by ESA and NASA.
Bertaux, J. L., \& Blamont, J. E. 1971, A\&A, 11, 200

1976, Nature, 262, 263

Bertaux, J. L., et al. 1997, Sol. Phys., 175, 737

Bleszynski, S. 1987, A\&A, 180, 201

Dalaudier, F., Bertaux, J. L., Kurt, V. G., \& Mironova, E. N. 1984, A\&A, 134,171

Flynn, B., Vallerga, J., Dalaudier, F., \& Gladstone, G. R. 1998, J. Geophys. Res., 103, 6483

Frisch, P. C. 1998, Space Sci. Rev., 86, 107

Gardner, L. D., et al. 1996, Proc. SPIE, 2831, 2 2000, Proc. SPIE, 4139, 362

Gloeckler, G., \& Geiss, J. 1998, Space Sci. Rev., 86, 127

Holzer, T. E. 1977, Rev. Geophys. Space Sci., 15, 467

Hovestadt, D., et al. 1995, Sol. Phys. 162, 441

Jelinsky, S. R., Siegmund, O. H., \& Mir, J. 1996, Proc. SPIE, 2808, 617

Judge, D. L., McMullin, D. R., \& Ogawa, H. S. 1999, J. Geophys. Res., 104,28321

Kohl, J. L., et al. 1995, Sol. Phys., 162, 313

Lallement, R., \& Bertin, P. 1992, A\&A, 266, 479

\section{REFERENCES}

Möbius, E., Rucinski, D., Hovestadt, D., \& Klecker, B. 1995, A\&A, 304, 505

Ogawa, H. S., Phillips, E., \& Judge, D. L. 1997, J. Geophys. Res., 102, 11557

Osantowski, J. F., Keski-Kuha, R. A. M., Herzig, H., Toft, A. R., Gum, J. S. \& Fleetwood, C. M. 1991, Adv. Space Res., 11, 185

Phillips, E., Judge, D. L., \& Carlson, R. W. 1982, J. Geophys. Res., 87, 1433

Rucinski, D., \& Fahr, H. J. 1989, A\&A, 224, 290

Rucinski, R., Cummings, A. C., Gloeckler, G., Lazarus, A. J., Möbius, E., \& Witte, M. 1996, Space Sci. Rev., 78, 73

Siegmund, O. H. W., et al. 1994, Proc. SPIE, 2280, 297

. 1995, Proc. SPIE, 2518, 344

Witte, M., Banaszkiewicz, M., \& Rosenbauer, H. 1996, Space Sci. Rev., 78, 289

Witte, M., Rosenbauer, H., Banaszkiewicz, M., \& Fahr, H. 1993, Adv. Space Res., 13, 121

Woods, T. N., Tobiska, W. K., Rottman, G. J., \& Worden, J. R. 2000, J. Geophys. Res., 105, 195 